\title{
Increased Fear Response to Contextual Cues in Mice Lacking the 5-HTIA Receptor
}

\author{
Kristen C Klemenhagen', Joshua A Gordon', Denis J David ${ }^{1,2}$, René Hen ${ }^{*, 1}$ and Cornelius T Gross ${ }^{1,3}$ \\ 'Center for Neurobiology and Behavior, Columbia University, New York, NY, USA; ${ }^{2}$ Laboratoire de Neuropharmacologie, EA 3544 MJENR, \\ Faculté de Pharmacie, Université Paris-Sud, Châtenay-Malabry, France; ${ }^{3}$ Mouse Biology Programme, European Molecular Biology Laboratory, \\ Monterotondo, Italy
}

\begin{abstract}
Serotonin IA receptor knockout (5-HTI AR KO) mice exhibit increased behavioral inhibition in conflict tests. To gain further insight into their anxiety-related phenotype, we subjected these mice to additional behavioral tests. First, we considered whether behavioral inhibition in these knockout mice is a consequence of reduced exploratory motivation. The knockout mice engage in normal exploration during a light-dark test and normal exploration of a novel object in a familiar environment, suggesting that the anxiety-related phenotype is not due to reduced exploratory drive. Second, we tested whether these mice exhibit increased behavioral inhibition in response to any aversive cues, or whether this response depends on cue modality. Knockout mice respond normally to discrete aversive cues in the Vogel lick-suppression test, arguing that their phenotype is restricted to conflict tests based on complex or spatial aversive cues. Third, to probe the processing of spatial aversive cues, we assessed fear conditioning to contextual cues. After contextual fear conditioning, knockout and wild-type (WT) mice express freezing responses when exposed to the training environment. However, when placed in an ambiguous environment containing both conditioned and novel cues, the freezing response of knockout mice does not significantly decrease as it does in WT mice, suggesting that the knockout fear response is biased toward threatening cues. We hypothesize that this inappropriate generalization of fearful behavior to a context containing both fearful and neutral stimuli, a phenomenon that occurs in a subset of human anxiety disorders such as panic disorder and post-traumatic stress disorder, underlies the anxiety phenotype of 5-HTIAR KO mice. Neuropsychopharmacology (2006) 3 I, I 0 I - I I I. doi: I 0. I 038/sj.npp. I 300774; published online 25 May 2005
\end{abstract}

Keywords: serotonin; knockout; behavior; anxiety; fear conditioning; G-protein-coupled receptor

\section{INTRODUCTION}

Anxiety is a mental state elicited in anticipation of threat. Feelings of anxiety are accompanied by behavioral and physiological responses that facilitate coping with danger, including avoidance and arousal. Behavioral inhibition, a response that is characterized by increased vigilance and heightened autonomic arousal in reaction to novel environments and stimuli, has been observed in anxious children (Kagan et al, 1988) and in rodent models of anxiety (Archer, 1973). In humans, the tendency or susceptibility to experience anxiety is called trait anxiety and is relatively constant over the lifetime (Spielberger, 1983).

Perturbations of two genes of the serotonergic system have been associated with increased anxiety. Polymorphisms in the promoter of the human serotonin transporter

*Correspondence: Dr R Hen, N.Y.S.P.I. Kolb Research Annex, Room 767, I05I Riverside Drive, Box 87, New York, NY 10032-2695, USA; Tel: + I 212543 5137, Fax: + I 2125435074 ,

E-mail: rh95@columbia.edu

Received 16 September 2004; revised 24 March 2005; accepted 19 April 2005

Online publication: 19 April 2005 at http://www.acnp.org/citations/ Npp041905040429/default.pdf and serotonin $1 \mathrm{~A}$ receptor that alter the transcriptional activity of these genes are associated with increased trait anxiety (Lesch et al, 1996; Greenberg et al, 2000; Lemonde et al, 2003; Strobel et al, 2003). Furthermore, knockout mice lacking the serotonin transporter or serotonin $1 \mathrm{~A}$ receptor exhibit increased anxiety-related behaviors (Parks et al, 1998; Ramboz et al, 1998; Heisler et al, 1998; Sibille et al, 2000; Gross et al, 2002; Pattij et al, 2002; Holmes et al, 2003). Interestingly, transient blockade of serotonin transporter or serotonin $1 \mathrm{~A}$ receptor function during early postnatal development mimics the adult knockout phenotype and argues that serotonin signaling during development plays a critical role in establishing normal anxiety behavior in adulthood (Ansorge et al, 2004; Gross et al, 2002). PET imaging in humans has also implicated disturbances of serotonin $1 \mathrm{~A}$ receptor function in the pathophysiology of panic disorder (PD) (Neumeister et al, 2004).

Our present work focuses on further characterizing serotonin $1 \mathrm{~A}$ receptor knockout (5-HT1AR KO) mice as a model of human anxiety. 5-HT1AR KO mice display reduced locomotion and increased avoidance of aversive areas in the open-field, elevated-plus maze, and elevatedzero maze tests (Parks et al, 1998; Ramboz et al, 1998; Heisler et al, 1998; Sibille et al, 2000), as well as increased 
latency to feed in a novel environment (Gross et al, 2002), all behavioral responses indicative of increased behavioral inhibition to aversive stimuli. In addition, knockout mice demonstrate increased tachycardia following exposure to a novel environment (Pattij et al, 2002), reflecting increased arousal to threatening stimuli.

We asked three questions about the phenotype of 5HT1AR KO mice. First, is the decreased locomotor behavior seen in response to novelty a consequence of a decrease in exploratory drive, or is this phenotype a reflection of increased behavioral inhibition? Second, do the knockout mice exhibit behavioral inhibition to simple aversive cues, or is their behavioral inhibition provoked only by complex aversive cues such as spatial novelty? Third, how do knockout and wild-type (WT) mice respond to an ambiguous environment containing both threatening and nonthreatening cues? Our findings demonstrate that 5HT1AR KO mice show increased behavioral inhibition specifically when faced with an environment containing complex and ambiguous threatening cues. Based on this result, these mice may serve as a model of the subset of human anxiety characterized by the generalization of anxiety responses to nonthreatening environments, including PD and post-traumatic stress disorder (PTSD).

\section{METHODS}

\section{Animals}

Adult male 5-HT1AR KO and age-matched WT mice were generated as described previously (Gross et al, 2002). Mice were housed in groups of 3-5, maintained on a $12 \mathrm{~h}$ light/ dark cycle (lights off at $1800 \mathrm{~h}$ ), and provided with standard laboratory food and water ad libitum, except where noted for the Vogel lick-suppression test. Animals were genotyped by PCR. All experiments were reviewed and approved by the institutional animal care and use committee of Columbia University and conducted in accordance with the Guide for the Care and Use of Laboratory Animals published by the US National Institutes of Health (revised, 1996).

\section{Light-Dark Test}

Testing was conducted in an open-field chamber measuring $43.2 \times 43.2 \mathrm{~cm}$ with a white floor and clear walls (Med Associates, Vermont, USA), with a dark plastic box insert opaque to visible light but transparent to infrared motion tracking beams covering half of the area of the open-field chamber. The tracking beams and data collection were controlled by a computer running the software Activity Monitor (Med Associates). Following the modifications proposed by Belzung et al (1987), the open-field box was divided into two equal areas with a doorway located in the center of the dark wall at floor level to allow passage between the light and dark chambers. The light compartment was brightly illuminated with an $8 \mathrm{~W}$ fluorescent tube (400 lx).

The test was performed in a quiet, darkened room. Mice were individually housed in cages containing a handful of bedding from their home cage and acclimated to the room at least $1 \mathrm{~h}$ before the test. Between each trial, the light and dark compartments were cleaned with disinfectant and allowed to dry. Naïve mice were placed individually in the testing chamber in the middle of the light area facing away from the doorway to the dark compartment. The test was $5 \mathrm{~min}$ in duration, and time spent in the dark and light compartments and the ambulatory distance were recorded by the computer. Sample sizes for this test were $10 \mathrm{WT}$ and $18 \mathrm{KO}$.

\section{Novel Object Recognition}

Testing occurred in a plastic box measuring $43.2 \times 43.2 \mathrm{~cm}$ with a white floor and clear walls. A pattern of black and white bars was affixed to one wall of the chamber during all sessions to provide proximal spatial cues. Owing to the relatively low baseline activity of our background strain, testing conditions were chosen to increase spontaneous locomotion and exploratory activity, as follows. All tests were conducted between 1800 and $2200 \mathrm{~h}$, during the dark cycle. Animals (16 WT, $14 \mathrm{KO}$ ) were transported to the testing room in groups of $2-4$, in cages containing bedding from their home cages and enclosed within a dark box to avoid exposure to bright light. The testing room was dimly lit by a $25 \mathrm{~W}$ light bulb and behavior sessions were recorded with an infrared-sensitive video camera. The chamber was conceptually divided into four quadrants (clockwise from upper left, A-D). Quadrants A, B, and C were occupied by an object during sessions $1-3$, while quadrant $D$ was left empty. To encourage exploration, the objects contained both textural and olfactory cues. The objects were $2-6 \mathrm{~cm}$ in height and $2-6 \mathrm{~cm}$ in width, and all objects contained the same base $(1.5 \mathrm{~cm}$ tall cylindrical section of a clear plastic $1.5 \mathrm{ml}$ microcentrifuge tube with $1 \mathrm{~mm}$ holes punched in the walls). A fresh $1 \mathrm{~cm}$ piece of incense was placed in the base of each object immediately prior to testing. Each object had a unique incense odor associated with it and consisted of the following textures: plastic pipette tips arranged with the small tip end arrayed in a circle parallel to the floor (object A), a smooth Pasteur pipette bulb (object B), curved metal paperclip pieces (object C), and strips of latex glove with ragged edges (novel object). Each object was affixed to the top of a microcentrifuge tube base with glue.

The mice were tested in six 5-min sessions separated by a 3-min delay, except for between sessions 3 and 4, where there was a 60 -min delay. Between sessions, the mice were returned to an individual cage containing bedding from their home cage. Sessions 1-3 consisted of habituation sessions containing a configuration of three objects in quadrant A, B, and C; quadrant D was left empty. During session 4 , the spatial novelty session, object B was moved to quadrant $\mathrm{D}$. Object $\mathrm{B}$ remained in quadrant $\mathrm{D}$ for the remainder of the test sessions. Before session 6 , the novel object session, object A was replaced with the novel object. At the beginning of each session, the animal was introduced into the testing chamber in the corner of quadrant $\mathrm{C}$, facing the center of the arena. The object in quadrant $\mathrm{C}$ remained constant through all six sessions.

All behavioral sessions were scored from videotape by an observer blind to genotype. Object exploration was scored as the number of visits to each object where a visit was defined as orientation of the animal toward the object with its nose within $2 \mathrm{~cm}$ of the object for a minimum of $1 \mathrm{~s}$. The chamber and objects were cleaned between animals with 
Roccal disinfectant (Pharmacia and Upjohn, Kalamazoo, $\mathrm{MI}$ ) and allowed to dry before a new mouse was introduced into the chamber.

\section{Vogel Lick-Suppression Test}

The Vogel drinking test was conducted in a behavioral chamber measuring $14 \times 15 \times 12 \mathrm{~cm}$ (MED Associates, Vermont, USA). The experimental protocol was similar to procedures described elsewhere (Vogel et al, 1971). Mice were deprived of water for $24 \mathrm{~h}$ before the test. During testing, the mouse was placed in the testing chamber and allowed to lick the waterspout 20 times to establish knowledge of the drinking spout location. Following the first 20 licks, a shock was delivered through the drinking spout at the completion of a run of 20 licks. The duration of the shock received depended on the amount of time the animal was in contact with the spout, but was limited to a maximum of $0.1 \mathrm{~s}$. Timing began after the initial 20 licks, and ended after $5 \mathrm{~min}$. During each session, a computer running MedPC (MED Associates, Vermont, USA) recorded the number of licks and controlled the delivery of shocks. The testing chamber was cleaned with disinfectant between mice. Shock intensities of 0.15 and $0.30 \mathrm{~mA}$ were used in separate experiments. All animals were tested only once. Sample sizes for each group were $0.15 \mathrm{~mA}$ : $16 \mathrm{WT}$ and 29 $\mathrm{KO}$; and $0.30 \mathrm{~mA}: 31 \mathrm{WT}$ and $27 \mathrm{KO}$.

\section{Fear Conditioning}

Training for all animals occurred in behavioral chambers (MED Associates, Vermont, USA) equipped to deliver a scrambled foot shock via the grid floor, measuring $21 \times 17 \times 21 \mathrm{~cm}$, and housed within soundproof wooden boxes. Each session was videotaped for later analysis. On training and testing days, the animals were transported to the behavioral room in their home cages and allowed to acclimate to the room for at least $45 \mathrm{~min}$ prior to introduction into the behavioral chambers. Freezing during the entire testing session was scored from videotape by an observer blind to the genotype and treatment of the animal.

Training. To establish a training context, the wire bars on the floor of the behavioral chamber were left exposed, and three drops of Roccal disinfectant were applied to paper toweling in the tray underneath the cage to provide an olfactory cue. The training session lasted $8 \mathrm{~min}$, with three $0.7 \mathrm{~mA}$ shocks delivered at 80,250 , and $360 \mathrm{~s}$ for a duration of $1 \mathrm{~s}$. Control animals were placed in the chamber for $8 \mathrm{~min}$, but received no shocks. After training, the animals were placed in holding cages containing a handful of bedding from their home cage, and all animals were returned to their home cage in the animal colony at the conclusion of the training sessions.

Testing in ambiguous environment. At $24 \mathrm{~h}$ after training, freezing behavior was assessed in a similar environment in one group of animals ( $37 \mathrm{WT}, 35 \mathrm{KO}$ ). The ambiguous environment was identical to the training environment, except that it had a smooth white plastic sheet on the floor to cover the metal bars and was scented with three drops of $70 \%$ ethanol. The animals were placed in the chamber and their freezing behavior was assessed for $4 \mathrm{~min}$. After testing, the animals were placed in holding cages containing a handful of bedding from their home cage, and all animals were returned to their home cage in the animal colony at the conclusion of the testing sessions.

Testing in training environment. At $24 \mathrm{~h}$ following testing in the ambiguous context, the same animals were returned to the training context and freezing was assessed for $4 \mathrm{~min}$. After testing, the animals were placed in holding cages containing a handful of bedding from their home cage, and all animals were returned to their home cage in the animal colony at the conclusion of the testing sessions.

Testing in novel environment. A separate group of animals (20 KO, $20 \mathrm{WT}$ ) were subjected to the same training procedure and tested for $4 \mathrm{~min}$ in a novel environment $24 \mathrm{~h}$ later. Like the ambiguous environment, the novel environment differed from the training environment by odor cue $(70 \%$ ethanol vs Roccal) and tactile cue (white plastic sheet on the floor $v s$ exposed bars). In addition, the testing occurred in a different shaped testing chamber $(14 \times 15 \times 12 \mathrm{~cm}$; MED Associates, Vermont, USA $)$ with a different visual cue on the wall (colored box $v s$ stripes). The chamber was not enclosed in a soundproof box, was exposed to ambient fluorescent lighting, and was located in a different testing room. Additionally, there were distal cues visible on the walls of the testing room, and the animals were transported from the colony to the novel testing room on a different cart. The experimenter also wore nitrile gloves for handling (latex was used in the training and ambiguous contexts), wore white clothing (dark colors were worn in the other contexts), and wore plastic shoe covers (paper shoe covers were used in the other contexts).

Extinction testing. At $24 \mathrm{~h}$ after testing in the training context, the first group of animals (37 WT, $35 \mathrm{KO}$ ) was again returned to the training context and freezing was assessed for $4 \mathrm{~min}$. This testing was repeated at 24-h intervals for a total of 10 days. After testing, the animals were placed in holding cages containing a handful of bedding from their home cage, and all animals were returned to their home cage in the animal colony at the conclusion of the testing sessions.

Shock threshold testing. After fear conditioning and extinction, the group of $37 \mathrm{WT}$ and $35 \mathrm{KO}$ animals were tested to determine the minimum shock intensity required to evoke flinching, movement, jumping, and vocalization responses. No differences were detected between animals that had previously been exposed to shock and those that had been assigned to the control group (data not shown), so the data from both types of animal was pooled. Intensities tested were $0.05,0.1,0.2,0.3,0.4,0.5,0.6,0.7$, and $1.0 \mathrm{~mA}$. Each mouse was subjected to five shocks, in increasing order of strength, presented at unpredictable intervals ranging from 30 to $60 \mathrm{~s}$ with a duration of $1 \mathrm{~s}$. The presence or absence of behaviors evoked by each shock intensity were recorded by an observer blind to genotype and previous treatment. 


\section{RESULTS}

\section{Light-Dark Test}

5-HT1AR KO mice show increased behavioral inhibition in several tests of anxiety-related behavior that depend on novel spatial cues to elicit defensive behaviors. In this test, we sought to determine whether KO mice have a deficit in exploratory behavior that might explain their increased behavioral inhibition under these conditions and to test whether the KO mice show increased avoidance of the aversive light compartment. We calculated the time spent in the light compartment out of the total testing time (300s) (Figure 1a). Two outliers in the KO group that were greater than 2 SDs from the mean were excluded from the analysis (values: 12.4 and $18 \mathrm{~s}$ in the dark compartment). KO mice spent significantly less time in the light compartment than WT mice $(\mathrm{t}[24]=2.0639$, $p=0.015$, two-tailed $t$-test). Overall exploration was measured by comparing the distance traveled during the test (Figure 1b). KO and WT mice did not differ in this measure $(\mathrm{t}[24]=2.0639, p=0.691$, NS, two-tailed $t$-test $)$. Furthermore, the number of transitions was not significantly different between $\mathrm{KO}$ and WT mice $(\mathrm{t}[24]=1.6252$, $p=0.117$, NS, two-tailed $t$-test, data not shown). As transitions are reported to habituate over time, this parameter is thought to reflect exploratory activity (Bourin and Hascoët, 2003). These results demonstrate that $\mathrm{KO}$ and WT mice do not differ in their exploratory drive, but $\mathrm{KO}$ mice have greater avoidance of the aversive light side of the testing chamber and spend more time in the dark chamber.

\section{Object Exploration Test}

To further examine the exploratory drive of the KO animals under different conditions, we subjected WT and KO mice to an object exploration test. During six 5-min, closely spaced sessions in a novel open arena, mice were exposed to
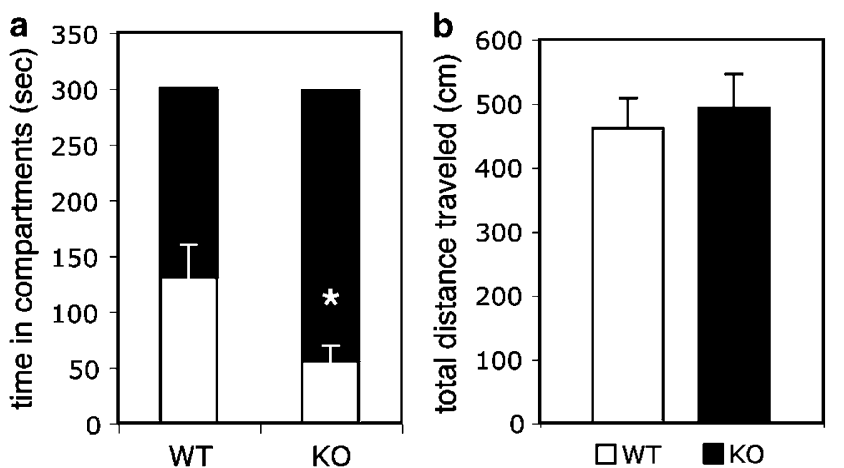

Figure I Compartment preference and exploratory behavior in the light-dark test. Data are expressed as the mean \pm SEM. (a) Time spent in the light $(\square)$ and dark $(\square)$ compartments during the 5-min test period. $\mathrm{KO}$ mice spent significantly less time in the aversive light compartment compared to the WT mice $(t[24]=2.0639, p=0.015$, two-tailed $t$-test). (b) Total exploratory activity during the test. $\mathrm{KO}$ and WT mice did not significantly differ in their total amount of exploration during the test $(t[24]=2.0639, p=0.691, N S$, two-tailed $t$-test). Sample size: WT $=10$ and $\mathrm{KO}=16$. three objects of distinct textural and olfactory identity (see Methods for details). Initially, each of three quadrants contained a distinct object $(\mathrm{A}, \mathrm{B}$, and $\mathrm{C})$, and the fourth quadrant was empty (D). Between sessions 3 and 4 , the object in quadrant $\mathrm{B}$ was moved to quadrant $\mathrm{D}$ to provide a new spatial arrangement of objects without changing their identity. Such manipulations have been shown to elicit hippocampal-dependent increases in exploration specific to the displaced object (Save et al, 1992; Mumby et al, 2002). Between sessions 5 and 6 , the object in quadrant $A$ was replaced by a novel object. In this design, we sought to test whether a novel object would elicit exploratory behavior in a familiarized environment.

Visits to the object in quadrant A (which is replaced by a novel object in session 6), visits to the spatial displacement object in quadrants $\mathrm{B}$ and $\mathrm{D}$, and visits to the control object in quadrant $C$ were plotted over the six sessions (Figure $2 \mathrm{a}-\mathrm{c}$ ). Figure $2 \mathrm{a}$ shows visits to the object in quadrant $\mathrm{A}$ during sessions 5 and 6 , and repeated measures ANOVA analysis finds a significant main effect of session $(F[1,28]=8.987, p=0.006)$, no main effect of genotype $(\mathrm{F}[1,28]=2.346, p=0.137)$, and no session $\times$ genotype interaction $(\mathrm{F}[1,28]=1.027, p=0.320)$ between sessions 5 and 6 , demonstrating similar responses to novelty in WT and KO mice in a familiarized environment. Newman-Keuls planned post hoc comparisons of visits to the object in quadrant A revealed that $\mathrm{KO}$ mice significantly increased exploration in session 6 compared to session $5(p<0.05$; Figure 2a). A smaller increase in visits was seen in WT mice, but did not reach significance. Furthermore, this increase in visits was specific to the quadrant where the novel object was introduced, since neither genotype increased exploration in session 6 in quadrant $\mathrm{C}$ (Figure 2c), the control quadrant that contained the same object in all sessions (Newman-Keuls, $p>0.05, \mathrm{NS})$.

Unexpectedly, neither genotype showed significant increases in exploratory activity in response to object displacement from quadrant $\mathrm{B}$ to quadrant $\mathrm{D}$ during session 4 (Figure 2b). Repeated measures ANOVA analysis of visits during sessions 3 and 4 showed a main effect of genotype $(\mathrm{F}[1,28]=7.869, p=0.009)$, no main effect of session $(\mathrm{F}[1,28]=0.729, \quad p=0.401)$, and no session $\times$ genotype interaction $(\mathrm{F}[1,28]=0.007, p=0.932)$, suggesting that in this testing protocol, displacement of an object under these testing conditions was not sufficient to elicit increased exploratory activity.

Quadrant C contained a control object that remained the same through all six sessions and was used as a measure of habituation (Figure 2c). As expected, KO mice made fewer visits to the control object during the initial sessions, reflecting increased behavioral inhibition in a novel environment. Repeated measures ANOVA analysis showed significant main effects of genotype $(F[1,28]=9.990$, $p=0.0038)$ and session $(\mathrm{F}[5,140]=3.227, p=0.0087)$, as well as a significant genotype $\times$ session interaction $(\mathrm{F}[5,140]=2.882, p=0.0165)$. Fisher's PLSD post hoc tests demonstrate fewer average visits to the two objects by $\mathrm{KO}$ mice in sessions 1 and $2\left(P_{1}=0.0115 ; P_{2}=0.0032\right.$; see Figure $2 \mathrm{c})$ until session $3\left(P_{3}=0.0694\right)$ when the animals became habituated to the objects and the arena, and WT and $\mathrm{KO}$ exploration was similar. Therefore, object novelty 
results in sessions 5 and 6 should not be affected by the genotype differences in object exploration when the environment is novel.
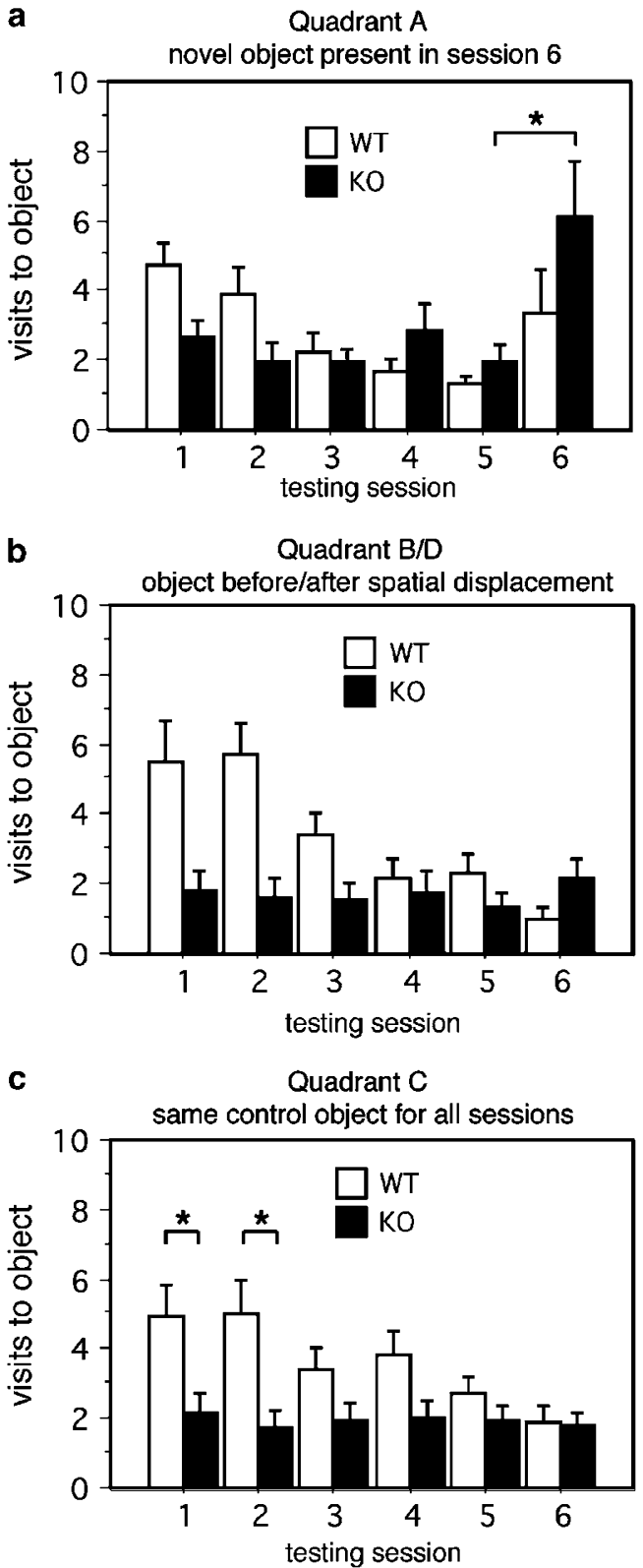

Figure 2 Visits to objects in an arena. (a) Visits to the object in quadrant A. During session 6 , the familiar object in quadrant $A$ was replaced by a novel object. ANOVA analysis of activity in sessions 5 and 6 shows a significant effect of session evoked by the novel object, but no significant effects of genotype, and no genotype $\times$ session interaction, suggesting that WT and $\mathrm{KO}$ animals show equal increased exploration of the novel object. NewmanKeuls planned post hoc comparisons show that the $\mathrm{KO}$ mice explore the object in quadrant A significantly more when it is novel $(* p<0.05)$, whereas the increase in exploration of the WT animals does not reach significance. (b) Visits to the spatial novelty object, before and after displacement. Repeated measures ANOVA showed no increase in exploration of the object between sessions 3 and 4 , the spatial novelty session. ANOVA main effect of session was nonsignificant $(F[1,28]=0.729, p=0.40 I$, NS). (c) Visits to the control object. Although there is a significant effect of genotype in the first two sessions $\left(P_{1}=0.0115 ; P_{2}=0.0032\right)$, the WT and $K O$ mice habituate to similar levels of exploration by session $3\left(P_{3}=0.0694\right)$. Sample size: $\mathrm{WT}=16$ and $\mathrm{KO}=14$.

\section{Vogel Lick-Suppression Test}

To determine whether increased behavioral inhibition in $\mathrm{KO}$ mice is also seen in response to simple, discrete aversive cues, we tested WT and KO mice in the Vogel licksuppression test, a benzodiazepine-sensitive test of behavioral inhibition (Vogel et al, 1971; Millan, 2003). In the Vogel lick-suppression test, drinking behavior motivated by a $24 \mathrm{~h}$ water deprivation is suppressed by the delivery of a mild shock to the animal via the drinking spout following every 20 licks. The total number of licks completed in $5 \mathrm{~min}$ can be readily quantified and reflects behavioral inhibition induced by the aversive stimulus.

We tested licking behavior in WT and KO mice under two shock intensities $(0.15$ and $0.30 \mathrm{~mA})$ to assess their threshold for shock-induced behavioral inhibition. To control for their motivation to drink, we also included a group of mice that did not receive shock. Figure 3 shows the total licks performed during the 5-min test period. At $0.15 \mathrm{~mA}$, ANOVA revealed no significant main effect of treatment $(\mathrm{F}[1,41]=3.012, p=0.090)$ or genotype $(\mathrm{F}[1,41]=3.196$, $p=0.081$ ). However, at $0.30 \mathrm{~mA}$, ANOVA demonstrated a main effect of treatment $(\mathrm{F}[1,54]=43.167, p<0.0001)$ and no effect of genotype $(\mathrm{F}[1,54]=0.573, p=0.452)$. Fisher's PLSD post hoc test revealed a significant effect of treatment for both WT $(p<0.0001)$ and KO $(p=0.0002)$ animals. These findings demonstrate that the threshold for behavioral inhibition in this test does not differ between WT and KO mice.

\section{Contextual Fear Conditioning - Identical Environment}

In order to better understand the capacity of KO mice to process spatial aversive cues and to investigate possible cognitive contributions to the anxiety-related phenotype of 5-HT1AR KO mice, we assessed fear conditioning to contextual cues. Fear conditioning was induced by delivering a mild inescapable foot shock to WT and KO mice in a novel environment with specific tactile, olfactory, and visual

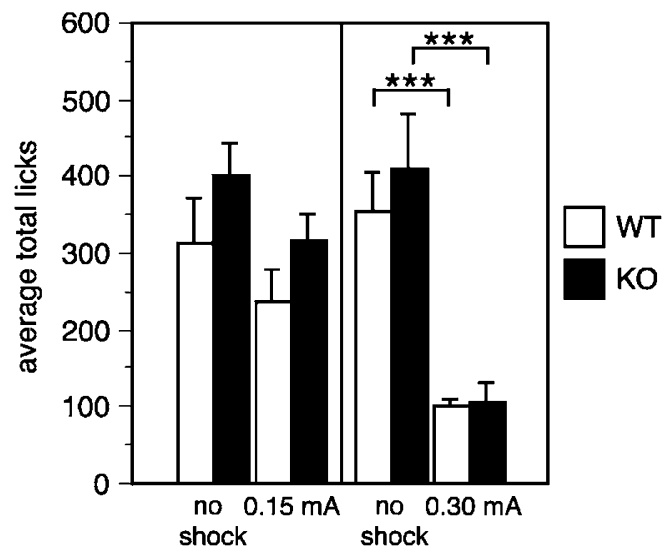

Figure 3 Vogel lick-suppression test. At the $0.15 \mathrm{~mA}$ shock dosage, there were no significant differences in the number of licks for WT and $\mathrm{KO}$ mice between shock and control groups. At $0.30 \mathrm{~mA}$, both $\mathrm{WT}$ and $\mathrm{KO}$ mice in the shock group licked the spout significantly less than nonshock controls (Fisher's PLSD post hoc test, ***WT: $p<0.000 \mathrm{I}$; ****KO: $p=0.0002$ ). Sample size, $0.15 \mathrm{~mA}: W T=16$ and $K O=29$. Sample size, $0.30 \mathrm{~mA}: \mathrm{WT}=31$ and $\mathrm{KO}=27$. 
cues. Freezing behavior during re-exposure to the identical training context $48 \mathrm{~h}$ later was used as a measure of contextual conditioning (Figure 4). Mice receiving no foot shock were included to control for differences in baseline freezing behavior. Exploratory ANOVA analysis in the identical context revealed main effects of treatment $(\mathrm{F}[1,68]=71.793, \quad p<0.0001)$ and genotype $(\mathrm{F}[1,68]=$ $5.818, p=0.019)$, and no significant genotype $\times$ treatment interaction $(\mathrm{F}[1,68]=1.481, p=0.228)$. Tukey-Kramer tests were used to focus on pairwise planned comparisons of treatment and genotype effects (Keppel and Wickens, 2004). Freezing behavior was elevated in both WT and KO mice compared to their respective nonshock controls, and in the identical context shocked KO mice showed greater levels of freezing than shocked WT mice (TukeyKramer tests significant at the 0.05 level). These results demonstrate that $\mathrm{KO}$ mice are not impaired in their ability to associate aversive stimuli with complex spatial cues.

\section{Contextual Fear Conditioning-Ambiguous Environment}

As the tests in which KO mice have previously shown increased anxiety-related behavior are based on situations where the animal must make a choice between behavioral responses to weakly aversive and strongly aversive stimuli, we reasoned that a fear conditioning phenotype might only be revealed in these mice when they were challenged with conflicting or ambiguous contextual cues. We tested this hypothesis by measuring freezing behavior of WT and KO mice in an ambiguous similar context containing both conditioned and novel cues (Figure 4). The similar testing context was identical to the training context, except that tactile and olfactory cues were novel (see Methods for details). Initial ANOVA analysis of this context reveals main effects of treatment $(F[1,68]=63.611, p<0.0001)$ and genotype $(\mathrm{F}[1,68]=15.621, p=0.0002)$, as well as a treatment $\times$ genotype interaction $\quad(\mathrm{F}[1,68]=6.519$, $p=0.013)$. Tukey-Kramer tests indicate that KO mice continue to freeze significantly more than WT animals in the shock treatment group $(p<0.05)$. Furthermore, a planned comparison across the identical and similar contexts shows that while shocked WT animals significantly decrease their level of freezing in the similar context, shocked $\mathrm{KO}$ animals do not modify their level of freezing (Tukey-Kramer, $p<0.05$ and Tukey-Kramer NS at the 0.05 level), suggesting that when challenged with ambiguous cues, KO mice have a bias toward responding to conditioned cues.

\section{Contextual Fear Conditioning - Novel Environment}

In order to demonstrate that the increased freezing behavior of KO mice in the ambiguous similar context was dependent on the presence of conditioned cues, we also examined freezing behavior of WT and KO mice in an entirely novel context after contextual fear conditioning (Figure 4). Initial ANOVA analysis in the novel context revealed a main effect of treatment $(\mathrm{F}[1,36]=13.949, p=0.001)$, but not genotype $(\mathrm{F}[1,36]=1.293, \quad p=0.263) \quad$ or treatment $\times$ genotype $(\mathrm{F}[1,36]=0.848, p=0.363)$. Follow-up Tukey-

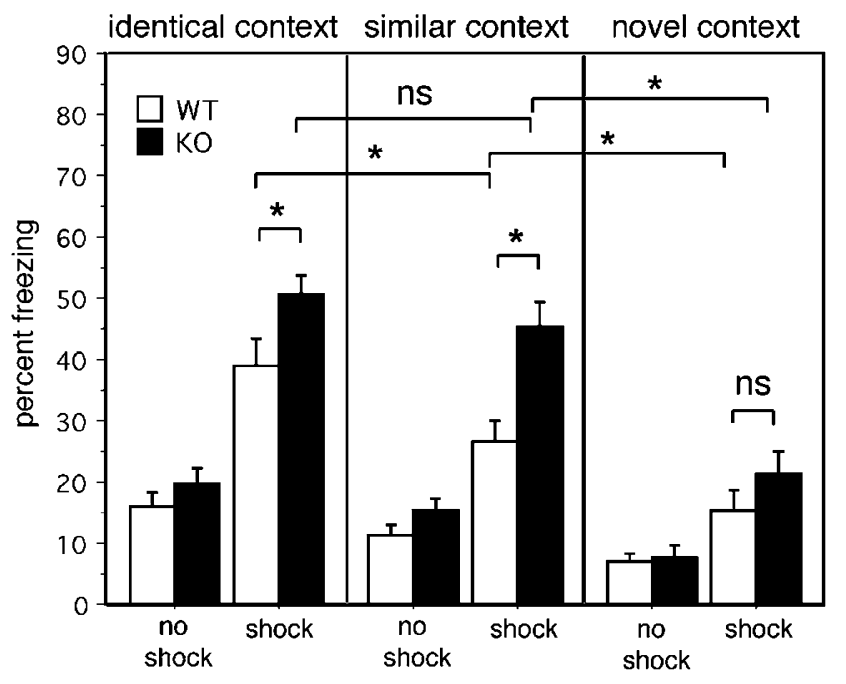

Figure 4 Contextual fear conditioning. Freezing was assessed in the identical (training) context, an ambiguous context similar to the training context, and a novel context. In the training context, both WT and KO mice showed increased freezing compared to nonshock control groups, and shocked $\mathrm{KO}$ mice froze significantly more than WT mice. In a similar context, where tactile and olfactory cues were novel, but spatial cues from the training context were still present, the $\mathrm{KO}$ mice froze significantly more than the WT mice, and maintained their high level of freezing similar to the training context, whereas WT froze significantly less than in the training context (Tukey-Kramer tests at $p<0.05$ level). In a novel context where many cues were changed from the training environment, both genotypes exhibited low levels of freezing, differed from their respective levels of freezing in the similar context, and did not differ significantly by genotype within the context. All * significant at $p<0.05$, Tukey-Kramer test. Sample size, identical and similar: $W T=37$ and $K O=35$. Sample size, novel: $\mathrm{WT}=20$ and $\mathrm{KO}=20$.

Kramer planned comparisons showed that there was no significant difference in freezing level between shocked WT and $\mathrm{KO}$ animals, and there was a significant decrease in freezing for both shocked WT and KO animals compared to the behavior of their respective shocked WT and KO groups in the similar context $(p<0.05)$, confirming that increased freezing of the $\mathrm{KO}$ in the ambiguous similar context is dependent on conditioning to specific cues in the training context.

\section{Contextual Fear Conditioning - Extinction}

The increased freezing behavior of the KO mice in the ambiguous context could be the result of having reached the ceiling of their response range through an overall increase in their efficacy of contextual conditioning. In order to test for the saturation of conditioning efficacy in KO mice, we examined extinction to contextual fear conditioning. Increased conditioning efficacy should be revealed during extinction as a prolongation of high levels of freezing behavior. We exposed WT and $\mathrm{KO}$ mice to the training context for $4 \mathrm{~min}$ each day over a period of 10 days. Freezing was scored during the re-exposure on days $1,2,3$, 4, 5, and 10 (Figure 5). Repeated measures ANOVA analysis showed main effects of treatment $(F[1,67]=28.850$, $p<0.0001)$, genotype $(\mathrm{F}[1,67]=5.249, p=0.0251)$, day $(\mathrm{F}[5,355]=44.455, p<0.0001)$, and interactions of day $\times$ 


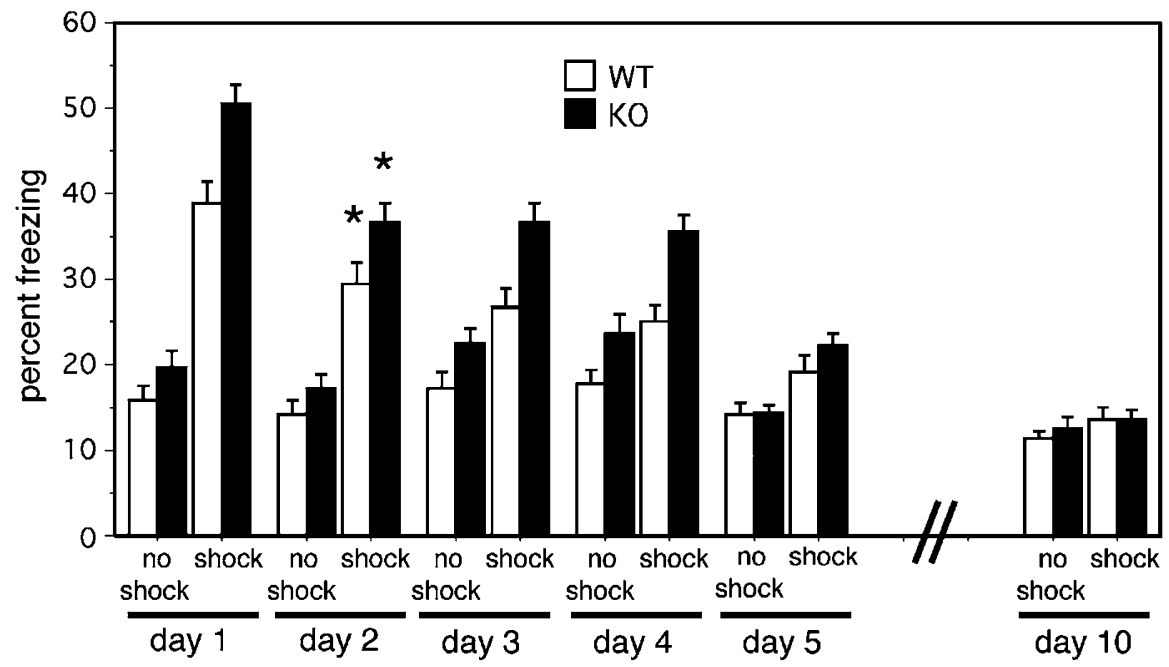

Figure 5 Extinction of fear conditioning. Freezing during re-exposure to the training context is shown for days I, 2, 3, 4, 5, and I0. Day I corresponds to the identical context panel in Figure 4, the session when the animals were first re-exposed to the training context. There is no significant genotype difference in the rate of extinction (ANOVA day $\times$ treatment $\times$ genotype $(F[5,355]=0.490, p=0.7836, N S)$, demonstrating that extinction of contextual fear conditioning does not differ between WT and KO mice. Furthermore, both genotypes significantly reduced their freezing between days I and 2 (TukeyKramer, $\left.{ }^{*} p<0.05\right)$, showing that the freezing response was not saturated in either genotype. Sample size: $\mathrm{WT}=37$ and $\mathrm{KO}=35$.

treatment $(\mathrm{F}[5,355]=19.973, p<0.0001)$ and day $\times$ genotype $(F[5,355]=2.982, \quad p=0.0119)$, but no significant interaction of day $\times$ treatment $\times$ genotype $(\mathrm{F}[5,355]=0.490$, $p=0.7836)$. Tukey-Kramer test comparisons of freezing levels between days 1 and 2 for the KO and WT shock treatment groups show a significant decrease in freezing between the days for both groups $(p<0.05)$. These results demonstrate that extinction to contextual fear conditioning does not differ between WT and KO mice, and argue that the fear conditioning response of $\mathrm{KO}$ and WT mice to contextual cues was not saturated in this test.

\section{Shock Threshold Test}

To investigate whether WT and KO mice exhibit differences in their reactivity to foot shock, we quantified the threshold required to elicit four different behavioral responses to foot shock: flinching, movement, vocalization, and jumping. The minimum shock intensity required to elicit each behavior in WT and KO mice is shown in Figure 6. Repeated measures ANOVA indicated no main effect of genotype $(\mathrm{F}[1,70]=$ $0.858, p=0.358)$ and no interaction between behavior and genotype $(\mathrm{F}[3,210]=1.759, p=0.156)$.

\section{DISCUSSION}

5-HT1AR KO mice exhibit increased anxiety-related behaviors in conflict tests, including the open-field, elevatedplus maze, elevated-zero maze, and novelty-suppressed feeding paradigms. This phenotype is described as avoidance, a form of behavioral inhibition that serves as a model of human anxiety with face and predictive validity (Kagan et al, 1988; Hirshfeld et al, 1992; Graeff et al, 1998). To better understand the origins of this phenotype, we have assessed WT and KO mice in additional behavioral tests. Our results suggest that the KO phenotype is not due to a

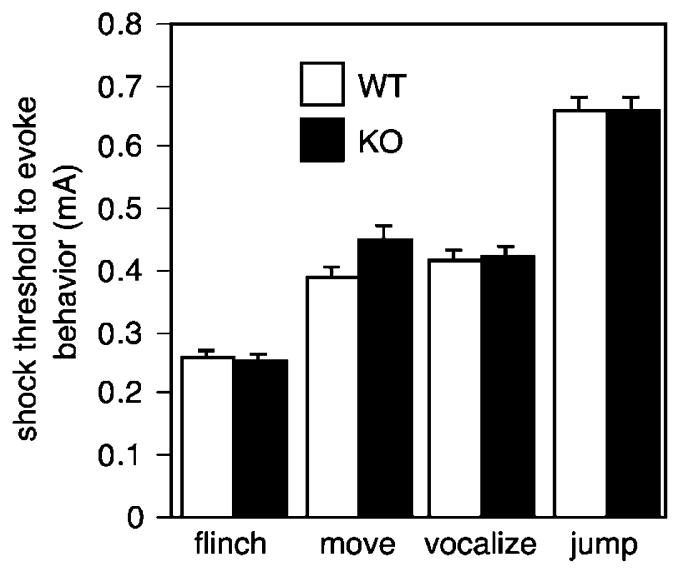

Figure 6 Shock reactivity thresholds. The shock dosage required to evoke four behaviors (flinch, movement, vocalization, and jump) was measured. No significant effect of genotype was detected by ANOVA $(F[1,70]=0.858, p=0.358, N S)$, suggesting that $W T$ and $K O$ mice are not differentially sensitive to foot shock. Sample size: $W T=37$ and $K O=35$.

deficit in exploratory drive, and argue that the $\mathrm{KO}$ mice display increased behavioral inhibition in comparison to WT mice when challenged with a threatening context. This behavior may be the result of a bias in the processing of threatening stimuli that leads to inappropriate generalization of fear responses.

\section{Normal Responses to Novelty and Discrete Aversive Cues}

Behavioral inhibition observed in conflict tests may be the consequence of a general reduction in motivation to explore. We tested this hypothesis by examining levels of exploratory activity in the light-dark test (Figure 1). The light-dark test allows measurement of both spontaneous 
exploratory activity and avoidance of an aversive, brightly lit chamber. Naïve mice prefer the dark chamber, and the time spent in the light is a measure of anxiety-like behavior (Hascoët et al, 2001). In this test, WT and KO mice exhibited equal levels of exploration. However, KO mice spent significantly less of the testing period in the light chamber compared to the WT mice. These results indicate that when given the choice to explore in light and dark chambers, the exploratory drive of the KO and WT mice are equal, but the KO spend a greater proportion of their time in the less aversive dark chamber.

Exploration elicited by a novel object in a familiar environment was examined in the novel object exploration test (Figure 2). Following habituation to objects in an open field, WT and KO mice made similar numbers of visits to the familiar objects. Replacement of a familiar object with a novel object elicited similar increases in exploratory behavior in WT and KO mice, suggesting that KO mice do not have a general reduction in levels of motivation to explore. Displacement of a familiar object failed to elicit a significant increase in exploration of the displaced object in either the KO or WT groups. As this test failed to measure an increased response to the displaced object in the WT group, it is possible that the testing protocol was not optimized to detect a reaction to a subtle change in object configuration in terms of the time between testing sessions or other features of the testing protocol. It would be interesting to pursue this possibility in future experiments with $\mathrm{KO}$ mice, since reaction to a spatially displaced object as been reported to be a hippocampal-mediated task (Save et al, 1992; Mumby et al, 2002). Finally, a post hoc comparison indicating increased visits to the novel object reveals that $\mathrm{KO}$ mice may be more responsive to novel cues in a habituated environment.

We next asked whether the increased avoidance behavior of KO mice was limited to spatial aversive cues, or whether similar increases in avoidance behavior could be elicited in response to nonspatial aversive cues. We find that $\mathrm{KO}$ mice perform normally in the Vogel lick-suppression test, an amygdala-dependent conflict test that is based on discrete, nonspatial threatening cues and is sensitive to benzodiazepine anxiolytics (Figure 3; Vogel et al, 1971; Yamashita et al, 1989; Millan, 2003). The normal response of KO mice in this test argues that KO mice exhibit a specific subtype of anxiety-related behavior characterized by excessive behavioral inhibition to complex spatial aversive cues. Furthermore, cued fear conditioning, another amygdala-dependent task, is normal in 5-HT1AR KO mice (Groenink et al, 2003), suggesting that perturbation of 5-HT1AR function in the amygdala in the $\mathrm{KO}$ mice does not play a major role in establishing their behavioral inhibition phenotype.

\section{Increased Behavioral Inhibition to an Ambiguous Environment}

We used contextual fear conditioning to examine the response of $\mathrm{KO}$ mice to aversive spatial cues in more detail. WT and KO mice exhibited contextual fear conditioning, showing significant freezing responses compared to nonshock control groups during re-exposure to the training context after $48 \mathrm{~h}$ (Figure 4). Low levels of freezing behavior were also seen in both genotypes during exposure to a novel context with a large number of cues that were different from the training context. When tested in an ambiguous similar environment containing a mix of novel and conditioned cues, both WT and KO mice froze significantly more than in the novel context, indicating that both groups of mice discriminated between the threat level of the similar and novel contexts. When freezing is compared across the identical training and similar contexts, WT mice freeze significantly less in the similar context than in the identical context, whereas KO mice show similar levels of freezing behavior and do not discriminate between the identical and similar contexts in their freezing response. These data demonstrate that, unlike WT mice, KO mice can use a subset of the conditioned cues to recall the aversive association; or alternatively, in an ambiguous environment KO mice attend better or respond more strongly to threatening cues. This behavior is reminiscent of symptoms associated with PTSD and PD, where an overgeneralization process occurs when specific trauma or panic-related cues trigger symptoms in an otherwise neutral environment (Gorman et al, 2000; Marshall and Garakani, 2002). Hypervigilance for threatening cues seen in PTSD and PD patients may also be consistent with the trend observed in our KO mice for increased exploration of a novel object and the significantly higher levels of freezing seen in the KO mice compared to the WT mice within the identical and similar contexts.

An alternative interpretation of these data is that $\mathrm{KO}$ mice express greater freezing behavior in the ambiguous context because they make a stronger association between the context and the aversive stimulus during training. The levels of freezing in both WT and KO mice compared to their nonshock control groups in the identical training context suggest that the WT and KO mice both successfully associate the aversive stimulus and the context. The extinction experiment also suggests that freezing was not saturated in the KO or the WT mice. We found no evidence that either group was conditioned to a ceiling level of freezing during the training and subsequent extinction, since both genotypes significantly decreased their freezing on the second day of exposure to the training environment (Figure 5). Furthermore, WT and KO mice show equal sensitivities to foot shock (Figure 6). These results support our interpretation that both WT and KO mice were conditioned to fear the training context, but that KO mice show greater anxiety-related responses when faced with threatening contextual cues.

\section{Role of the Hippocampus in the 5-HT1AR KO Anxiety Phenotype}

The observation that KO mice show increased anxietyrelated responses specifically when challenged with ambiguous and complex spatial cues suggests that a dysfunction in hippocampally mediated spatial processing may underlie their phenotype. Although defects in spatial memory have been reported in 5-HT1AR KO mice on a mixed SwissWebster;129/Sv genetic background (Sarnyai et al, 2000), spatial memory as measured in the Morris water maze is only modestly affected in the background used here (Wolff et al, 2004). Thus, while dramatic spatial navigation defects are not an essential part of the anxiety-related phenotype, 
subtle defects may reflect an underlying neural perturbation that affects both cognitive function and the processing of aversive stimuli. We have previously shown that expression of 5-HT1AR in the forebrain rescues the anxiety-related behavioral phenotype of $\mathrm{KO}$ mice (Gross et al, 2002). Within the forebrain, expression of the receptor is prominent in the CA1 region of the hippocampus, a structure important for the formation of complex sensory associations and implicated in the modulation of anxietyrelated behavior (Holland and Bouton, 1999; McNaughton and Gray, 2000). Lesions of the hippocampus decrease anxiety-related behavior in the elevated-plus maze (Kjelstrup et al, 2002) and the delivery of benzodiazepines to the hippocampus is anxiolytic (Menard and Treit, 1999). Furthermore, human imaging studies implicate the hippocampus and its surrounding input and output structures in the discrimination of contextual cues as well as the processing of aversive conditioned stimuli (Buchel et al, 1999; Bar and Aminoff, 2003). Finally, electrophysiological recordings in behaving animals show that the frequency of CA1 pyramidal neuron firing encodes information about the strength of conditioning to aversive stimuli (Munera et al, 2001), suggesting that cognitive circuits in the brain play a critical role in associative processing of aversive stimuli. Defects in hippocampal function at the cellular level have been documented in 5-HT1AR KO mice, including decreased paired pulse inhibition (Sibille et al, 2000) and increased basal excitability and dendritic arborization ( $\mathrm{R}$ Hen and J Monckton, personal communication) in CA1 pyramidal neurons. While further experiments are warranted to demonstrate the relevance of these physiological changes, it seems plausible that the hippocampus plays a central role in the anxiety-related phenotype seen in the KO.

\section{Role of the Hippocampus in Human Anxiety Disorders}

Generalization of a fear response to an ambiguous environment containing both threatening and nonthreatening contextual cues is a characteristic of PD and PTSD. Although the common and unique elements of pathophysiology underlying different human anxiety disorders are largely unknown, abnormalities of hippocampal structure and function have been observed in both PD and PTSD. Imaging studies of PD patients have consistently found abnormal bilateral asymmetries in cerebral blood flow and perfusion in the hippocampal areas (Reiman et al, 1984; Nordahl et al, 1990; De Cristofaro et al, 1993), as well as increased glucose metabolism (Bisaga et al, 1998). Imaging of GABA receptor binding revealed that a PD population had decreased benzodiazepine binding in the hippocampus (Bremner et al, 2000). Decreased binding of the 5-HT1AR has also been observed in PD patients in the cingulate cortex and midbrain raphe (Neumeister et al, 2004), but this effect has not been observed in the hippocampus; the 5HT1AR is widely expressed in the brain and it is possible that 5HT1AR dysfunction in multiple areas of the brain may contribute to anxiety.

Numerous studies have correlated decreased hippocampal volume with PTSD populations that suffered prolonged exposure to trauma, such as combat experience and childhood abuse (Bremner et al, 1995, 1997; Gurvits et al, 1996; Stein et al, 1997). Recent evidence suggests that decreased hippocampal volume is a predictor of vulnerability to develop PTSD, rather than a consequence of PTSD-related stress (Gilbertson et al, 2002). The role of hippocampal atrophy in PTSD is not yet understood (Sapolsky, 2002), but a study of long-term SSRI treatment of PTSD patients suggests that boosting levels of serotonin can lead to modest increases in hippocampal volume (Vermetten et al, 2003).

The results presented in this study implicate perturbation of 5-HT1AR function in the phenomenon of inappropriate generalization of fear to a nonthreatening environment in 5HT1AR KO mice, and given that the anxiety-like behaviors are specific to complex spatial threat cues, we hypothesize that the anxiety-like phenotype is a consequence of 5HT1AR dysfunction in the hippocampus. Taken together with the emerging knowledge of the role of serotonergic activity and the hippocampus in human anxiety, these results suggest that the 5-HT1AR KO mouse may serve as a useful model for a subset of anxiety disorders characterized by hypervigilance for threat cues and generalization of anxiety behavior to nonthreatening environments.

\section{ACKNOWLEDGEMENTS}

This study was supported by NIH Grants F32 MH12364 and K01 MH64948 (CTG), NIMH Grant R01 MH068542, NIH Grant P01 MH48125 and a NARSAD Distinguished Investigator Award (RH), and NIMH Grant K08 MH069823 (JAG). KCK was supported by an NSF Graduate Research Fellowship. JAG is a Pfizer Foundation Postdoctoral Fellow in Biological Psychiatry, and is supported by the Bowman Family Foundation as a NARSAD Young Investigator. We are grateful to Caroline Ling for her help with initial fear conditioning experiments.

\section{REFERENCES}

Ansorge MS, Zhou M, Lira A, Hen R, Gingrich JA (2004). Early-life blockade of the 5-HT transporter alters emotional behavior in adult mice. Science 306: 879-881.

Archer J (1973). Tests for emotionality in rats and mice: a review. Anim Behav 21: 205-235.

Bar M, Aminoff E (2003). Cortical analysis of visual context. Neuron 38: 347-358.

Belzung C, Misslin R, Vogel E, Dodd RH, Chapouthier G (1987). Anxiogenic effects of methyl-beta-carboline-3-carboxylate in a light/dark choice situation. Pharmacol Biochem Behav 28: 29-33.

Bisaga A, Katz JL, Antonini A, Wright CE, Margouleff C, Gorman JM et al (1998). Cerebral glucose metabolism in women with panic disorder. Am J Psychiatry 155: 1178-1183.

Bourin M, Hascoët M (2003). The mouse light/dark box test. Eur J Pharmacol 463: 55-65.

Bremner JD, Innis RB, White T, Fujita M, Silbersweig D, Goddard AW et al (2000). SPECT [I-123] iomazenil measurement of the benzodiazepine receptor in panic disorder. Biol Psychiatry 47: 96-106.

Bremner JD, Randall P, Scott TM, Bronen RA, Seibyl JP, Southwick $\mathrm{SM}$ et al (1995). MRI-based measurement hippocampal volume patients with combat-related posttraumatic stress disorder. Am J Psychiatry 152: 973-981.

Bremner JD, Randall P, Vermetten E, Staib L, Bronen RA, Mazure $C$ et al (1997). Magnetic resonance imaging-based measurement of hippocampal volume in posttraumatic stress disorder related 
to childhood physical and sexual abuse - a preliminary report. Biol Psychiatry 41: 23-32.

Buchel C, Dolan RJ, Armony JL, Friston KJ (1999). Amygdalahippocampal involvement in human aversive trace conditioning revealed through event-related functional magnetic resonance imaging. J Neurosci 19: 10869-10876.

De Cristofaro MT, Sessarego A, Pupi A, Biondi F, Faravelli C (1993). Brain perfusion abnormalities in drug-naive, lactatesensitive panic patients: a SPECT study. Biol Psychiatry 33: 505-512.

Gilbertson MW, Shenton ME, Ciszewski A, Kasai K, Lasko NB, Orr SP et al (2002). Smaller hippocampal volume predicts pathologic vulnerability to psychological trauma. Nat Neurosci 5: 1242-1247.

Gorman JM, Kent JM, Sullivan GM, Coplan JD (2000). Neuroanatomical hypothesis of panic disorder, revised. Am J Psychiatry 157: 493-505.

Graeff FG, Netto CF, Zangrossi Jr H (1998). The elevated T-maze as an experimental model of anxiety. Neurosci Biobeh Rev 23: 237-246.

Greenberg BD, Li Q, Lucas FR, Hu S, Sirota LA, Benjamin J et al (2000). Association between the serotonin transporter promoter polymorphism and personality traits in a primarily female population sample. Am J Med Genet 96: 202-216.

Groenink L, Pattij T, De Jongh R, Van der Gugten J, Oosting RS, Dirks A et al (2003). 5-HT1A receptor knockout mice and mice overexpressing corticotropin-releasing hormone in models of anxiety. Eur J Pharmacol 463: 185-197.

Gross C, Zhuang X, Stark K, Ramboz S, Oosting R, Kirby L et al (2002). Serotonin1A receptor acts during development to establish normal anxiety-like behaviour in the adult. Nature 416: 396-400.

Gurvits TV, Shenton ME, Hokama H, Ohta H, Lasko NB, Gilbertson MW et al (1996). Magnetic resonance imaging study of hippocampal volume in chronic combat-related posttraumatic stress disorder. Biol Psychiatry 40: 1091-1099.

Hascoët M, Bourin M, Dhonnchadha BA (2001). The mouse lightdark paradigm: a review. Prog Neuropsychopharmacol Biol Psychiatry 25: 141-166.

Heisler LK, Chu HM, Brennan TJ, Danao JA, Bajwa P, Parsons LH et al (1998). Elevated anxiety and antidepressant-like responses in serotonin 5-HT1A receptor mutant mice. Proc Natl Acad Sci USA 95: 15049-15054.

Hirshfeld DR, Rosenbaum JF, Biederman J, Bolduc EA, Faraone SV, Snidman N et al (1992). Stable behavioral inhibition and its association with anxiety disorder. J Am Acad Child Adolesc Psychiatry 31: 103-111.

Holland PC, Bouton ME (1999). Hippocampus and context in classical conditioning. Curr Opin Neurobiol 9: 195-202.

Holmes A, Yang RJ, Lesch KP, Crawley JN, Murphy DL (2003). Mice lacking the serotonin transporter exhibit 5-HT(1A) receptor-mediated abnormalities in tests for anxiety-like behavior. Neuropsychopharmacology 28: 2077-2088.

Kagan J, Reznick JS, Snidman N (1988). Biological bases of childhood shyness. Science 240: 167-171.

Keppel G, Wickens TD (2004). Design and Analysis: A Researcher's Handbook, 4th edn. Pearson Education Inc.: Upper Saddle River, NJ. pp 111-131.

Kjelstrup KG, Tuvnes FA, Steffenach HA, Murison R, Moser EI, Moser MB (2002). Reduced fear expression after lesions of the ventral hippocampus. Proc Natl Acad Sci USA 99: $10825-10830$.

Lemonde S, Turecki G, Bakish D, Du L, Hrdina PD, Bown CD et al (2003). Impaired repression at a 5-hydroxytryptamine $1 \mathrm{~A}$ receptor gene polymorphism associated with major depression and suicide. J Neurosci 23: 8788-8799.

Lesch KP, Bengel D, Heils A, Sabol SZ, Greenberg BD, Petri S et al (1996). Association of anxiety-related traits with a polymorph- ism in the serotonin transporter gene regulatory region. Science 274: $1527-1531$.

Marshall RD, Garakani A (2002). Psychobiology of the acute stress response and its relationship to the psychobiology of posttraumatic stress disorder. Psychiatr Clin N Am 25: 385-395.

McNaughton N, Gray JA (2000). Anxiolytic action on the behavioural inhibition system implies multiple types of arousal contribute to anxiety. J Affect Disord 61: 161-176.

Menard J, Treit D (1999). Effects of centrally administered anxiolytic compounds in animal models of anxiety. Neurosci Biobehav Rev 23: 591-613.

Millan MJ (2003). The neurobiology and control of anxious states. Prog Neurobiol 70: 83-244.

Mumby DG, Gaskin S, Glenn MJ, Schramek TE, Lehmann H (2002). Hippocampal damage and exploratory preferences in rats: memory for objects, places, and contexts. Learn Mem 9: 49-57.

Munera A, Gruart A, Munoz MD, Fernandez-Mas R, DelgadoGarcia JM (2001). Hippocampal pyramidal cell activity encodes conditioned stimulus predictive value during classical conditioning in alert cats. J Neurophysiol 86: 25712582.

Neumeister A, Bain E, Nugent AC, Carson RE, Bonne O, Luckenbaugh DA et al (2004). Reduced serotonin type 1A receptor binding in panic disorder. J Neurosci 24: 589-591.

Nordahl TE, Semple WE, Gross M, Mellman TA, Stein MB, Goyer P et al (1990). Cerebral glucose metabolic differences in patients with panic disorder. Neuropsychopharmacology 3: 261-272.

Parks CL, Robinson PS, Sibille E, Shenk T, Toth M (1998). Increased anxiety of mice lacking the serotonin1A receptor. Proc Natl Acad Sci USA 95: 10734-10739.

Pattij T, Groenink L, Hijzen TH, Oosting RS, Maes RA, van der Gugten J et al (2002). Autonomic changes associated with enhanced anxiety in 5-HT(1A) receptor knockout mice. Neuropsychopharmacology 27: 380-390.

Ramboz S, Oosting R, Amara DA, Kung HF, Blier P, Mendelsohn M et al (1998). Serotonin receptor 1A knockout: an animal model of anxiety-related disorder. Proc Natl Acad Sci USA 95: 14476-14481.

Reiman EM, Raichle ME, Butler FK, Herscovitch P, Robins E (1984). A focal brain abnormality in panic disorder, a severe form of anxiety. Nature 310: 683-685.

Sapolsky RM (2002). Chickens, eggs and hippocampal atrophy. Nat Neurosci 5: 1111-1113.

Sarnyai Z, Sibille EL, Pavlides C, Fenster RJ, McEwen BS, Toth M (2000). Impaired hippocampal-dependent learning and functional abnormalities in the hippocampus in mice lacking serotonin(1A) receptors. Proc Natl Acad Sci USA 97: 1473114736.

Save E, Poucet B, Foreman N, Buhot MC (1992). Object exploration and reactions to spatial and nonspatial changes in hooded rats following damage to parietal cortex or hippocampal formation. Behav Neurosci 106: 447-456.

Sibille E, Pavlides C, Benke D, Toth M (2000). Genetic inactivation of the serotonin(1A) receptor in mice results in downregulation of major $\mathrm{GABA}(\mathrm{A})$ receptor alpha subunits, reduction of GABA(A) receptor binding, and benzodiazepine-resistant anxiety. J Neurosci 20: 2758-2765.

Spielberger CD (1983). Manual for the State-Trait Anxiety Inventory (Form V). Consulting Psychologists Press: Palo Alto, CA.

Stein MB, Koverola C, Hanna C, Torchia MG, McClarty B (1997). Hippocampal volume in women victimized by childhood sexual abuse. Psychol Med 27: 951-959.

Strobel A, Gutknecht L, Rothe C, Reif A, Mössner R, Zeng Y et al (2003). Allelic variation in 5-HT1A receptor expression is associated with anxiety- and depression-related personality traits. J Neural Transm 110: 1445-1453. 
Vermetten E, Vythilingam M, Southwick SM, Charney DS, Bremner JD (2003). Long-term treatment with paroxetine increases verbal declarative memory and hippocampal volume in posttraumatic stress disorder. Biol Psychiatry 54: 693-702.

Vogel JR, Beer B, Clody DE (1971). A simple and reliable conflict procedure for testing anti-anxiety agents. Psychopharmacologia 21: 1-7.
Wolff M, Costet P, Gross C, Hen R, Segu L, Buhot M-C (2004). Agedependent effects of serotonin-1A receptor gene deletion in spatial learning abilities in mice. Brain Res Mol Brain Res 130: 39-48.

Yamashita K, Kataoka Y, Shibata K, Ozaki T, Miyazaki A, Kagoshima M et al (1989). Neuroanatomical substrates regulating rat conflict behavior evidenced by brain lesioning. Neurosci Lett 104: 195-200. 